\title{
Giving birth and becoming a parent during the COVID-19 pandemic: a qualitative analysis of 827 women's responses to an online survey in Norway
}

Tine S. Eri ( $\nabla$ tines@oslomet.no )

Oslo Metropolitan University

Soo Downe

University of Central Lancashire

Carina Vedeler

Oslo Metropolitan University

Ellen Blix

Oslo Metropolitan University

Anne Britt Vika Nilsen

Western Norway University of Applied Sciences

\section{Research Article}

Keywords: Pregnancy, labour, birth, qualitative study, COVID-19 pandemic, maternity care, post-partum care, experiences

Posted Date: May 12th, 2021

DOl: https://doi.org/10.21203/rs.3.rs-452774/v1

License: (c) (1) This work is licensed under a Creative Commons Attribution 4.0 International License. Read Full License 


\section{Abstract}

Background: When Europe was hit by the COVID-19 pandemic, changes were made in maternal care to reduce infections. In hospital maternity wards, postnatal wards, and neonatal units' companions and visitors were restricted. There is emerging evidence that changes in maternity care delivery and restrictions due to social distancing have been distressing for many childbearing women. We aimed to explore the experiences of giving birth and becoming a parent in Norway during the COVID-19 pandemic.

Methods: The study is based on the responses from women who provided in-depth qualitative comments to Babies Born Better survey version 3, December 2020. The responses were analysed with inductive thematic analysis.

Results: In all, 827 women who gave birth in 42 of 45 available birthing units across Norway, regardless of parity and mode of birth, were included. The qualitative analysis resulted in four themes: 1) Waiting period filled with stress and worries; 2) Feeling lonely, isolated and disempowered without the partner; 3 ) Sharing experiences and becoming a family; and 4) Busy postnatal care without compassion.

Conclusion: The COVID-19 pandemic has had an impact on women's experiences of giving birth and becoming a parent in Norway. The denial of companionship and postnatal visiting varied between hospitals, which suggests that some of the restrictions were related to organisational protectionism than the protection of women and babies. Early postnatal care is the area of care that seems to have been most negatively affected and clearly requires more attention. The balance between humanised healthcare and protection from infection has not been well maintained through this current pandemic.

\section{Background}

Childbirth is both a clinical episode and an existential life-event. The WHO Framework for improving quality of care for women during childbirth states that women's experience of care is just as important as clinical care provision (1). In their most recent intrapartum guidelines, the WHO recognises that a positive childbirth experience is significant to both the short- and long-term wellbeing of women and their infants (2). The guidelines highlight the importance of woman-centred care to optimise the experience of labour and childbirth for women and their babies by means of a holistic, human rights-based approach. To achieve this, quality healthcare systems must be built on respect and understanding and provide care tailored to women's circumstances and needs (3). Globally, women's perspectives of what constitutes respectful care is fairly consistent, and one important domain is ensuring access to family attendance and the presence of a labour companion of their choice (4). Having a companion present during labour and childbirth is also an important component of a positive birth experience, as well as being associated with a range of clinical benefits $(5,6)$.

When Europe was hit by the COVID-19 pandemic, changes were made in maternal care to reduce infections (7). Lockdowns and social distancing became common strategies, and the health services were reorganised to minimise the spread of the virus. Restrictions were imposed in hospital maternity 
wards, postnatal wards and neonatal units in relation to companions and visitors. There is emerging evidence that changes in care provision and restrictions due to social distancing have been distressing for many childbearing women, in Norway as in other countries, in addition to the general fear that can arise in the context of a pandemic $(8,9)$. A number of studies have reported on the psychological impact of being pregnant and becoming a parent during the pandemic. A Spanish study, for instance, that included 162 women found that women who gave birth during this period have suffered higher levels of stress, rated the quality of their care more poorly and more often suffered from postpartum depression compared to women who gave birth before the pandemic (10). A cross-sectional study that includes 9,041 women from Ireland, Norway, Switzerland, the Netherlands and the UK found high levels of depressive symptoms and generalised anxiety among pregnant and breastfeeding women during the pandemic (8). Two other studies found a rise in psychosocial problems in postnatal women associated with social distancing and restrictions in the UK and Italy $(11,12)$. A Swedish longitudinal study of 6,941 pregnant women and their partners showed that pregnant women experienced a dramatic increase in health-related worries during the pandemic (13).

There is nonetheless limited knowledge about the experience of giving birth and becoming a parent during the pandemic, and the potential impact of changes brought about by COVID-19 in the provision of healthcare on this experience. An Australian study found that women perceived having a baby to be an isolating experience (14), and a study performed in the UK showed that $39 \%$ of the 1,365 respondents reported changes to their birth plans due to COVID-19. The main changes reported were changes to the women's preferred place of birth and restrictions on the presence of a birth partner (15).

The aim of the present study was to explore experiences of giving birth and becoming a parent in Norway during the COVID-19 pandemic, based on the responses of a large sample of women who provided indepth qualitative comments on a European-wide birth experiences survey launched in June 2020.

\section{Material And Methods}

Data collection: We used data from the Babies Born Better (B3) Survey, Version 3. The B3 Survey is a mixed-methods web-based questionnaire designed to identify factors that underpin women's experiences of maternity care in Europe. The B3 project was developed within the framework of an EU COST networking Action (IS0907), "Childbirth Cultures, Concerns and Consequences: Creating a dynamic EU framework for optimal maternity care" and continued in Action IS 1405 (BIRTH).

This version of the survey was launched internationally through social media in June 2020 and is still open for responses. Women were eligible for inclusion if they had given birth during the last three years. The questionnaire comprised 23 questions with sub-questions (see Additional file 1). The first section requires fixed responses related to demographics and clinical factors. The subsequent two sections invite open responses, designed to elicit respondents' views of positive factors and suggestions for change after their experience of care. The answers to the following open-ended questions in these two sections formed the basis of this study: 
- "In the place where you gave birth, what were the three most positive experiences of your care?" "What do you think could have made your experience better?" (Question 17)

- "Imagine you are talking to a very close friend or family member who is pregnant, and that she is trying to decide where to give birth to her baby. She asks you what you think about the place you gave birth. Please answer here by finishing one or both of the following sentences: I think you should give birth at the place where I did because... I think you should not give birth at the place where I did because..." (Question 18)

- "Please write any comments you want to make here. These could explain your answers in more detail or add any other information you would like us to know about your experiences with maternity care" (Question 21)

Sampling: All respondents who had given birth in Norway from 1 March to 31 December 2020 and had given at least one response to question Q17, Q18 or Q21 were eligible for inclusion in this study. We excluded women who had answered the survey in a language other than Norwegian, Swedish, Danish or English (14 respondents).

Description of the data: Each woman could give up to a total of nine free-text responses to the openended questions; accordingly, the number of units of analysis were far higher than the number of respondents. The responses varied from short sentences to longer narrative accounts.

Procedure for analysis: Descriptive statistics were used for the quantitative analyses. We performed an inductive thematic analysis inspired by Braun \& Clark (16). Initially, the whole dataset was read through several times to get a good sense of the content. In the next step, meaning units relevant to the research question were identified and coded. The initial codes were thereafter organised into main codes, and themes were subsequently generated. Seen as a whole, the four final themes provide a coherent account that represents the content of the data. Two of the authors performed the qualitative analysis and the final themes were discussed and decided on by the whole team.

\section{Results}

In all, 827 women's responses were included in this study. Most women (87.8\%) chose to respond to the Norwegian version of the survey, while $12.2 \%$ responded in English. The mean age was 29.8, (SD 4.7), and $45 \%$ of the women were primiparous and $55 \%$ multiparous. Forty-two of 45 available birthing units in Norway were represented. Eighty-six per cent of the respondents gave birth in a hospital, $8.7 \%$ in an alongside midwifery unit, $0.8 \%$ in a freestanding midwifery unit, $1.1 \%$ had a planned homebirth and 0.7 gave birth before they arrived at the planned place of birth. The proportion of vaginal births was $73.2 \%$, operative vaginal births $9.8 \%$, and caesarean Sect. $11.7 \%$.

The qualitative analysis resulted in four themes: 1) Waiting period filled with stress and worries; 2) Feeling lonely, isolated and disempowered without the partner; 3 ) Sharing experiences and becoming a family; and 4) Busy postnatal care without compassion. 


\section{Waiting period filled with stress and worries}

Many women felt that being pregnant during a pandemic was a stressful waiting period characterised by increased vulnerability and worries concerning what lied ahead of them. The lack of information and not knowing what restrictions would apply at the hospital in terms of companionship, was described as stressful, burdensome and extremely tough. Not knowing what to do or where to get updated information was described as something that took a lot of energy during the pregnancy.

All the insecurity surrounding the birth and that my husband could not be with me afterwards was scary and annoying and drained my energy before the birth.

The women's anxiety and worry about them or their partner getting COVID-19 during the pregnancy also caused them stress. They were afraid an infection could affect the unborn child and found it very difficult to think about the risk of giving birth alone, without their partner, in isolation. This led to them placing restrictions on how they and their partner could live their lives during the pregnancy through self-imposed quarantine. The women also worried about being infected by COVID-19 when staying in the hospital during labour and post-partum, and how such a situation could affect them and their baby.

It was difficult to be worried in advance about whether my husband might not be present during the childbirth if he caught a cold.

The women learned that the birthing units' restrictions during the pandemic permitted the partner to accompany the woman when she had reached active labour, which contributed to anxiety and insecurity during the pregnancy in case they needed care during the latent phase of labour. Some women had sought care later than they had planned after going into labour because they were afraid they would be alone without their partner. This resulted in chaotic births with the women fully dilated on admission, and they feared they would give birth at home or before arrival without assistance. Some women who wanted a check-up during early labour described difficulties in getting permission to be admitted to the hospital because of the strict rules, which led to feelings of uncertainty and insecurity in the first phase of labour.

As a first-time mother during the coronavirus pandemic, there was a lot of extra stress and nervousness. It's difficult to be at home and feel that you are unwanted in the hospital. I was 5-6 cm when I finally begged to come in for a check-up.

\section{Feeling lonely, isolated and disempowered without the partner}

The women described feeling alone during their pregnancy when their partner was not allowed to attend the antenatal care sessions. This made them sad and led to a feeling of being "on your own". Some women, especially if there were complications, had received a great deal of information during antenatal check-ups, and felt unable to absorb everything by themselves. At times, they had also needed to make 
important decisions about their pregnancy and birth without their partner, which led to them feeling lonely and powerless.

I think it's absolutely terrible that my husband can't be there with me for the ultrasounds during my pregnancy. We live in the same house and have no symptoms.

The feeling of loneliness and being "on your own" was reinforced by being alone at a time when they really needed someone. When they arrived at the hospital, their partner often had to wait either outside the entrance or in the car in the parking lot, until he/she was given permission to enter. The women described being met by guards at the entrance, and then having to find their way to the birthing unit on their own. Some women spent hours or days alone in the hospital before labour started due to complications or the need for an induction, which caused them to feel isolated, insecure and afraid. Having labour induced at a hospital meant that they had to spend the whole period until reaching active labour alone, rather than being at home with their partner during early labour if induction was not necessary. Some women described hours of unbearable pain from induced contractions, on their own without their partner to support them, which made them feel extremely lonely, helpless and angry.

My birth was induced due to rising blood pressure and low amniotic fluid. I was alone for a day and a halt in an observation ward before I came to the delivery room and was allowed to be with my husband. It was very hectic in the ward and I felt very alone.

The women saw their partner as the one person they could "lean on"; who knew them and their needs and wishes. They strongly express needing his/her support and help to cope with labour, also prior to the active phase, and called for it when it was lacking. Very many women expressed that the thing they would have wanted to change was to have their partner present during all stages of labour and at the postnatal ward. They reported feeling unsafe and even scared when they were alone without their partner. In those situations when it was possible to have their partner present, the women emphasised this as the best part of their care, and some express gratefulness to the staff who sometimes overlooked the restrictions and individualised their situation.

The midwife allowed my partner to stay the night until before 8 in the morning since the child was born in the middle of the night. He had to leave before the change of shifts, as this was not really allowed at the hospital.

The women's feelings of loneliness and isolation are even more prominent in their accounts of their stay at the postnatal ward. The fact that their partner could not be with them throughout the stay and that they were often not allowed to leave the room at all, gave rise to difficult emotions. It could also cause them to feel imprisoned when being left alone in a small, hot room. The women reported feeling "on their own" and questioned whether that kind of care was even safe. The respondents emphasised how much they missed practical and emotional support from their partners after the birth. They believed that their partner being allowed to stay with them and the baby in the postnatal ward would have been both good for them and very helpful for the staff. The women felt that the postnatal ward had been understaffed and pointed 
out that their partner could have helped out and relieved the midwives and nurses with practical tasks such as fetching food and drinks.

The birth was quick and, as expected, really terrible. But the midwife was good, and I felt relatively safe. The post-partum days were very lonely, and I longed for my husband. It was difficult to get help (get food, talk about the baby, get a smile from someone). I felt that the staff were avoiding me and no one asked how I was doing.

\section{Sharing experiences and becoming a family}

The women emphasised wanting to be together as a family during the whole maternity episode; through pregnancy, birth and the post-partum period. They perceived it as a very special period with precious moments for the couple and the family. The women wanted to take the first steps of parenthood together with their partner and spend time with the newborn baby as a family. They described sadness if they lost the possibility of experiencing the joy and excitement of the first few hours and days as a unit and of creating shared memories for the future. Some women continued to express grief, sorrow and loss months after the birth if they had not been able to share the first few hours or days together.

It was quite tragic that the father was not allowed to attend the birth which was an elective $C$-section. This makes the birth experience difficult to think back on. We never got to experience meeting the twins together, just one of us at a time.

To be together as a family also meant deciding for themselves who they were going to share the experience of the new baby with and when. For example, some women thought it was a shame that older siblings could not come to meet the baby straight away to share the moment together as a family, instead having to wait until the mother and baby arrived home. Some women described how this important family moment had been taken away from them, and they felt miserable about this loss.

I was induced and gave birth two days later. Went home after six days. For me as a mum and my family, it was absolutely awful. Not being able to share the joy, the scary experience, the ups and downs with my husband and children. It IS still a crisis. The fact that we could not share this together is really painful. It hurts so much that we could not be together when we needed it the most.

The strong wish for a family focus is very evident in the data; many women highlight the best part of their maternity care as when they were given an opportunity to spend time with their partner and the newborn together, and they express gratefulness about situations when this was facilitated - despite the restrictions.

\section{Busy postnatal care without compassion}

Many women reported feeling safe and well cared for by competent staff during the birth, despite their partner only being able to be present during the active stage of labour. This is in stark contrast to their experiences of postnatal care. 
The labour ward was very good, but the experience of the postnatal care overshadows most of it. I felt overlooked and not taken care of.

The women frequently pointed out that the postnatal ward was seriously understaffed, they felt that the midwives and nurses had very little time for them and that their needs had not been fulfilled. Since they were often not allowed to leave their room, and their partners were not allowed to stay, the women had to ask the staff for help with everything they needed. For some, this led to them feeling that they were a bother. For others, who were not able to get out of bed on their own after, for example, a caesarean section or to carry the crying baby themselves, it resulted in feelings of helplessness and sorrow. Many women felt overlooked and not seen as a person and called for more compassion in postpartum care.

Being abandoned and left to fend for myself with a newborn baby has been a very traumatic experience for me. My partner was not allowed to attend the postpartum ward due to corona, and the staff was even more overworked than usual. No one had time to help me with breastfeeding. I was clearly told that there were others who needed the staff more than me when I asked for help. I just felt like I was bothering the staff.

The women felt that the midwives and nurses had signalled that they were overloaded with tasks, and this made the atmosphere in the ward busy and tense. The staff was often characterised as doing the best they could in a very difficult time, but also as being brusque and not always polite, failing to demonstrate an understanding of the new mothers' situation. This was especially evident in connection with breastfeeding consultations, which were sometimes described as cold and insensitive. Many women

felt that they had been given very little help with breastfeeding, and that the one or two consultations they received were quick, abrupt and insensitive. Furthermore, there was a feeling of being overruled by the staff on decisions concerning the mother and the baby, for instance when it came to breastfeeding versus bottle-feeding or using a pacifier.

Some of the staff at the postnatal hotel created unnecessary stress and negative thoughts by being patronising and stressed out.

A few multiparous women had, in contrast to the above, enjoyed not having visitors in the postnatal ward. They felt that they had enjoyed a couple of days off, without any pressure to receive visitors, on legitimate grounds.

\section{Discussion}

The findings of our study show that women experienced giving birth and becoming a parent during the COVID-19 pandemic in Norway in a variety of manners. However, the changes in the organisation of maternity care appear to have had a profound effect on their experiences, as summarised in the four themes generated from the analysis. 
Many women reported feelings of insecurity, anxiety, stress and worries throughout their pregnancy. They did not know what to expect nor what or who would be available to them, either during antenatal checkups or at the place they were going to give birth. Furthermore, they were concerned about their own and their family's health and well-being. This is in line with other studies investigating women's experiences during the COVID-19 pandemic $(8,13,17,18)$. Self-reported worries and related feelings of anxiety during pregnancy are common even under normal circumstances $(19,20)$, but there is mounting evidence that the impact on women's psycho-social wellbeing has been elevated during the pandemic, which, in turn, can increase the risk of post-partum depression (12).

For many respondents, the insecurity of not knowing who would be with them led to fearful anticipation that they might not be able to have their partner present during labour and birth. In some cases, this led to the women delaying admission to hospital after they had gone into labour. Unplanned home births and births during transport can increase the risk of adverse outcomes and be stressful for those involved (21, 22). For the women in this study, the anticipatory fear of being alone and abandoned during the early phases of labour led to worries during transport and chaotic situations upon admission.

Most women in the study reported that they in one way or another were negatively affected by the restrictions imposed on companionship in their maternity care. In Norway, the birth companion is usually the woman's partner and co-parent of the child. Very many women felt lonely and abandoned during the time they spent without their partner. This was particularly evident in the accounts of women who were induced, or women who needed professional care in the latent phase of labour. Likewise, women reported being alone in the post-partum ward with their newborn child for much of the time. During the pandemic, the World Health Organisation and other national and international guidelines producers have stated that all childbearing women must have the opportunity to have a positive childbirth experience, including companionship (23). The emphasis that this should be throughout the whole of labour and birth has been strong in some policy documents, including those stemming from the UK, where this point was specifically emphasised by the RCOG/RCM COVID documents, to overcome the accounts of women being abandoned during the early phases of labour and during induction (24). However, the results from this study, and others that are beginning to emerge from different countries around the world, suggest that these strong and evidence-based statements have not always been put into practice.

For many women, the time they spent alone during labour and birth far exceeded the time they were accompanied by their partner. It is well documented that continuous support for women during childbirth is beneficial and may improve outcomes for women and infants alike (5). Childbirth is also a neuropsycho-social event, comprising a complex interplay between maternal experiences and neurohormonal events (25). The neurohormonal processes, in particular oxytocinergic mechanisms, not only control the physiological aspects of labour and birth, but also contribute to the subjective psychological experience of birth. The neurobiological processes induced by the release of endogenous oxytocin during birth influence maternal behaviour and feelings in order to facilitate birth (25). The release of oxytocin is promoted by a warm and positive environment and gentle touching of the skin. The lack of a supportive partner for shorter or longer periods during labour and birth might lead to a disturbance in these 
neurobiological processes, thereby missing out on some of the factors that contribute to an optimised process of labour and the first interaction between mother and baby. Our data also indicate that many women perceived the lack of companionship as a safety issue. Some differentiated between the impression of the staff available to them during labour and birth being skilled and providing safe care, and that of feeling unsafe, psychologically and emotionally, from being abandoned, alone and lonely during many hours of labour before they were permitted to have a companion. These experiences may have unknown consequences, given that a "sense of security" is an important aspect of childbirth care for women in Norway (26).

More profoundly, our findings also show that the notion of companionship is far more than being a supporter or even just being a visitor to the woman. In the Norwegian context and in the context of many other European countries, being accompanied through the labour and birth journey by the co-parent of the baby sets the stage for the rest of family life together. The women in the study expressed grief and sadness when they had to experience these important first hours and days of their new family life alone. Some couples, for example after a complicated birth, were unable to meet their baby together for the first time as a team. Very many women in the study experienced that the partner had been forced to leave a few hours after the birth of the child. The mother had then been alone with the newborn for most of the time spent in the postnatal ward, without the possibility of sharing their experiences and thus creating shared memories for the future with the co-parent. Shared remembering has important social benefits for couples, and remembering the past together plays a crucial role to couples' identities, plans and relationships (27). This finding is in line with a study performed in the UK during the pandemic, where women describe distress when their families missed out on experiences that could never be regained (15). In a family perspective, it is important to take care of the partner as well as the new mother during and after childbirth (26). The partners of pregnant women have worried more about their partner's and their child's health, as well as their own health, during the COVID-19 pandemic (13). Research concerning fathers' psychological health shows that the experience of unpredictable complications gives rise to the need for additional support (28), raising the question of whether the unpredictable nature of becoming parents during the COVID-19 pandemic might have similar consequences.

The findings of our study show that many women experienced postnatal care as busy, cold and lacking compassion. They reported feeling like a bother when they asked for help, and felt that the postnatal wards were critically understaffed. In many cases, the women were not allowed to leave their rooms at all, and due to the partner being forced to leave the postpartum ward, or only allowed to visit for a couple of hours a day, the staff had to help the new mothers with everything they needed. Some women had to wait for hours for a meal, or for painkillers if needed. Our findings indicate that individual breastfeeding support was one of the aspects of early postnatal care that suffered the most. Many women reported having very little breastfeeding support or none at all, and that the support they had received felt insensitive and abrupt. This is in line with a study from the UK, where breastfeeding support was found to be one of the biggest concerns for women who gave birth during the pandemic (18). Current evidence suggests that there is a low risk of mother-child transmission through breastfeeding, and immediate postpartum breastfeeding is recommended and supported (29). Other countries have found that the rate 
of breastfeeding has decreased during the COVID-19 pandemic (15), and it has been indicated that social isolation and resulting psychosocial stress can have an impact on bonding between mothers and their babies (30). In the early postpartum period, it is important to provide breastfeeding support tailored to the individual needs of the mother (31).

Many of the respondents described their birth care and care providers in a positive light and placed this experience in strong contrast to what they perceived as bad quality care in the postpartum ward. National surveys of women's experiences of the whole course of maternity care in Norway show that women are least satisfied with the quality of postpartum care compared to antenatal care and care during labour and birth, also before the pandemic $(32,33)$. The findings of the present study indicate that this has been further aggravated during the COVID-19 pandemic.

\section{Strengths And Limitations}

One strength of this study is the large sample of women who gave birth during the COVID-19 pandemic $(n=827)$. The data includes the women's own accounts and consists of rich and extensive descriptions of their experiences of the care they received at the place they gave birth. No limitations were placed on the length of responses. A further strength is the diverse sample of women, who gave birth across all birth settings and at nearly all 45 available birthing units in Norway. Three small units with less than 50 births per year were not represented. Furthermore, the sample comprises both primiparous and multiparous women, regardless of the mode of birth. Another strength of the study is the short time span of one to nine months from giving birth to providing a response to the survey, which may contribute to low recall bias.

However, a number of limitations need to be taken into consideration. Self-selection bias, which can be an issue in self-recruiting surveys, may have contributed to recruiting women who had experienced dramatic episodes or who wanted to promote certain views. On the other hand, the survey was not designed to explore experiences during the pandemic explicitly, so there were no questions relating to experiences of the COVID-19 virus. This might therefore have reduced this effect. The sample was restricted to women who responded in a Nordic language or English, and 14 respondents were excluded for this reason.

\section{Conclusion}

Based on this study, we conclude that the COVID-19 pandemic has had an impact on women's experiences of giving birth and becoming a parent in Norway. The restrictions placed on companionship by the healthcare facilities seem to have generated more risk than benefit and have seriously impacted a wide range of aspects related to women's experiences of pregnancy, labour and birth and the early postpartum period. Early postnatal care is the area of care that seems to have been most negatively affected and clearly requires more attention. The denial of companionship and postnatal visits varies between hospitals, which suggests that some of the restrictions were more related to organisational protectionism than the protection of women and babies. It is therefore argued that the balance between 
humanised healthcare and protection from infection has not been well maintained through the current pandemic.

\section{Declarations}

\section{Ethics approval and consent to participate}

Ethical approval was granted by the Ethics Committee of the University of Central Lancashire, UK (Ethics Committee BuSH 222, 22nd January 2014). The study was approved by the Norwegian Data Inspectorate (ref:60547/3/HJTIRH, $4^{\text {th }}$ September 2018), no further ethical clearance was necessary Regional Committees for Medical and Health Research Ethics (ref:2017/1582, 05 $5^{\text {th }}$ October 2017).

\section{Consent for publication}

This manuscript contains no details, images, or videos relating to an individual person or persons that would require consent for publication.

\section{Availability of data and materials}

The datasets used and analysed during the current study are available from the corresponding author on reasonable request.

\section{Competing interests}

The authors report no conflicts of interest.

\section{Funding}

There was no funding related to this study.

\section{Authors contributions}

The conception and design of the work: TSE, ABVN. Acquisition of data: TSE, EB, SD, CV, ABVN. Analysis and interpretation of data: TSE, ABVN. Drafting the work and revising it critically for important intellectual content: TSE, EB, SD, CV, ABVN.

\section{Acknowledgements}

This paper derives from the Babies Born Better project that was developed as part of the EU-funded COST Action IS0907, and continued in EU COST Action IS1405: BIRTH: "Building Intrapartum Research Through Health - an interdisciplinary whole system approach to understanding and contextualising physiological labour and birth" (http://www.cost.eu/COST_Actions/isch/IS1405), supported by the COST (European Cooperation in Science and Technology) Programme as part of EU Horizon 2020. The work of all those who contributed to developing and running the Babies Born Better Survey is acknowledged. Details of the 
project, the Steering Group, and the Country Coordinators can be found here: http://www.babiesbornbetter.org/about/

\section{References}

1. Tunçalp Ö, Were W, MacLennan C, Oladapo O, Gülmezoglu A, Bahl R, et al. Quality of care for pregnant women and newborns-the WHO vision. BJOG: An International Journal of Obstetrics \& Gynaecology. 2015;122(8):1045-9.

2. WHO. World Health Organization. Intrapartum care for a positive childbirth experience. Geneva: WHO; 2018.

3. Renfrew MJ, McFadden A, Bastos MH, Campbell J, Channon AA, Cheung NF, et al. Midwifery and quality care: findings from a new evidence-informed framework for maternal and newborn care. The Lancet. 2014;384(9948):1129-45.

4. Shakibazadeh E, Namadian M, Bohren M, Vogel J, Rashidian A, Nogueira Pileggi V, et al. Respectful care during childbirth in health facilities globally: a qualitative evidence synthesis. BJOG: An International Journal of Obstetrics \& Gynaecology. 2018;125(8):932-42.

5. Bohren MA, Hofmeyr GJ, Sakala C, Fukuzawa RK, Cuthbert A. Continuous support for women during childbirth. Cochrane Database of Systematic Reviews. 2017(7).

6. Bohren M, Berger B, Munthe-Kaas H, Tunçalp Ö. Perceptions and experiences of labour companionship: A qualitative evidence synthesis. Cochrane database of systematic reviews (Online). 2019;2019:CD012449.

7. Coxon K, Turienzo CF, Kweekel L, Goodarzi B, Brigante L, Simon A, et al. The impact of the coronavirus (COVID-19) pandemic on maternity care in Europe. Midwifery. 2020;88:102779.

8. Ceulemans M, Foulon V, Ngo E, Panchaud A, Winterfeld U, Pomar L, et al. Mental health status of pregnant and breastfeeding women during the COVID-19 pandemic-A multinational cross-sectional study. Acta Obstet Gynecol Scand. 2021.

9. Souto SPAd, Albuquerque RSd, Prata AP. Fear of childbirth in time of the new coronavirus pandemic. Rev Bras Enferm. 2020;73(Suppl 2):e20200551-e.

10. Mariño-Narvaez C, Puertas-Gonzalez JA, Romero-Gonzalez B, Peralta-Ramirez MI. Giving birth during the COVID-19 pandemic: The impact on birth satisfaction and postpartum depression. Int J Gynaecol Obstet. 2020.

11. Ostacoli L, Cosma S, Bevilacqua F, Berchialla P, Bovetti M, Carosso AR, et al. Psychosocial factors associated with postpartum psychological distress during the Covid-19 pandemic: a cross-sectional study. BMC Pregnancy Childbirth. 2020;20(1):703-.

12. Fallon V, Davies SM, Silverio SA, Jackson L, De Pascalis L, Harrold JA. Psychosocial experiences of postnatal women during the COVID-19 pandemic. A UK-wide study of prevalence rates and risk factors for clinically relevant depression and anxiety. J Psychiatr Res. 2021;136:157-66. 
13. Naurin E, Markstedt E, Stolle D, Enström D, Wallin A, Andreasson I, et al. Pregnant under the pressure of a pandemic: a large-scale longitudinal survey before and during the COVID-19 outbreak. European Journal of Public Health. 2020;31(1):7-13.

14. Sweet L, Bradfield Z, Vasilevski V, Wynter K, Hauck Y, Kuliukas L, et al. Becoming a mother in the 'new' social world in Australia during the first wave of the COVID-19 pandemic. Midwifery. 2021:102996.

15. Vazquez-Vazquez A, Dib S, Rougeaux E, Wells JC, Fewtrell MS. The impact of the Covid-19 lockdown on the experiences and feeding practices of new mothers in the UK: Preliminary data from the COVID19 New Mum Study. Appetite. 2021;156:104985.

16. Braun V, Clarke V. Using thematic analysis in psychology. Qual Res Psychol. 2006;3:77 - 101.

17. Walsh S, Simmons-Jones F, Best R. Care during covid-19: partner attendance at maternity services. BMJ. 2020;371:m3973-m.

18. Karavadra B, Stockl A, Prosser-Snelling E, Simpson P, Morris E. Women's perceptions of COVID-19 and their healthcare experiences: a qualitative thematic analysis of a national survey of pregnant women in the United Kingdom. BMC Pregnancy Childbirth. 2020;20(1):600-.

19. PeÑAcoba-Puente C, Monge FJC, Morales DM. Pregnancy worries: a longitudinal study of Spanish women. Acta Obstet Gynecol Scand. 2011;90(9):1030-5.

20. Dennis C-L, Falah-Hassani K, Shiri R. Prevalence of antenatal and postnatal anxiety: Systematic review and meta-analysis. Br J Psychiatry. 2017;210(5):315-23.

21. Gunnarsson B, Smárason AK, Skogvoll E, Fasting S. Characteristics and outcome of unplanned outof-institution births in Norway from 1999 to 2013: a cross-sectional study. Acta Obstet Gynecol Scand. 2014;93(10):1003-10.

22. Engjom HM, Morken N-H, Høydahl E, Norheim OF, Klungsøyr K. Increased Risk of Peripartum Perinatal Mortality in Unplanned Births Outside an Institution: A Retrospective Population-Based Study. Obstetrical \& Gynecological Survey. 2018;73(1):8-11.

23. WHO. Every woman's right to a companion of choice during childbirth 2020a [Available from: https://www.who.int/news/item/09-09-2020-every-woman-s-right-to-a-companion-of-choice-duringchildbirth.

24. RCOG. Coronavirus (COVID-19) Infection in Pregnancy (version 13). London; 2021.

25. Olza I, Uvnas-Moberg K, Ekström-Bergström A, Leahy-Warren P, Karlsdottir SI, Nieuwenhuijze M, et al. Birth as a neuro-psycho-social event: An integrative model of maternal experiences and their relation to neurohormonal events during childbirth. PLOS ONE. 2020;15(7):e0230992.

26. Vedeler C, Nilsen ABV, Blix E, Downe S, Eri TS. What women emphasise as important aspects of care in childbirth: An online survey study Under review for BJOG: An International Journal of Obstetrics and Gynecology. 2020.

27. Harris CB, Barnier AJ, Sutton J, Keil PG. Couples as socially distributed cognitive systems: Remembering in everyday social and material contexts. Memory Studies. 2014;7(3):285-97. 
28. Vallin E, Nestander H, Wells MB. A literature review and meta-ethnography of fathers' psychological health and received social support during unpredictable complicated childbirths. Midwifery. 2019;68:48-55.

29. WHO. Frequently asked questions: Breastfeeding and COVID-19 for health care workers. . Journal of Human Lactation. 2020b;3(3):392-6.

30. Tscherning C, Sizun J, Kuhn P. Promoting attachment between parents and neonates despite the COVID-19 pandemic. Acta Paediatrica. 2020;109(10):1937-43.

31. Swerts $M$, Westhof $E$, Lemiengre J, Bogaerts $A$. The supporting role of the midwife during the first 14 days of breastfeeding: A descriptive qualitative study in maternity wards and primary healthcare. Midwifery. 2019;78:50-7.

32. Sjetne IS, Iversen HH, Kjøllesdal JG. A questionnaire to measure women's experiences with pregnancy, birth and postnatal care: instrument development and assessment following a national survey in Norway. BMC Pregnancy and Childbirth. 2015;15(1):182.

33. Holmboe O, Sjetne IS. Brukererfaringer med fødsels- og barselomsorgen i 2017. Nasjonale resultater [ User experiences of birth and postnatal care in 2017. National results]. Oslo: Institute of Public Health; 2018.

\section{Supplementary Files}

This is a list of supplementary files associated with this preprint. Click to download.

- BabiesBornBetterSurveyversion3.pdf 